\title{
Adsorption of P103 Nanoaggregates on Graphene Oxide Nanosheets: Role of Electrostatic Forces in Improving Nanosheet Dispersion
}

DOI:

10.1021/acs.langmuir.0c03206

\section{Document Version}

Accepted author manuscript

Link to publication record in Manchester Research Explorer

Citation for published version (APA):

Patil, R., Ray, D., Aswal, V. K., Bussy, C., Bahadur, P., \& Tiwari, S. (2021). Adsorption of P103 Nanoaggregates on Graphene Oxide Nanosheets: Role of Electrostatic Forces in Improving Nanosheet Dispersion. Langmuir, 37(2), 867-873. https://doi.org/10.1021/acs.langmuir.0c03206

\section{Published in:}

Langmuir

\section{Citing this paper}

Please note that where the full-text provided on Manchester Research Explorer is the Author Accepted Manuscript or Proof version this may differ from the final Published version. If citing, it is advised that you check and use the publisher's definitive version.

\section{General rights}

Copyright and moral rights for the publications made accessible in the Research Explorer are retained by the authors and/or other copyright owners and it is a condition of accessing publications that users recognise and abide by the legal requirements associated with these rights.

\section{Takedown policy}

If you believe that this document breaches copyright please refer to the University of Manchester's Takedown Procedures [http://man.ac.uk/04Y6Bo] or contact uml.scholarlycommunications@manchester.ac.uk providing relevant details, so we can investigate your claim.

\section{OPEN ACCESS}




\title{
Adsorption of P103 nanoaggregates over graphene oxide nanosheets: role of electrostatic
}

forces

\author{
Rahul Patil ${ }^{\mathrm{a}}$, Debes Ray ${ }^{\mathrm{b}}$, Vinod K. Aswal ${ }^{\mathrm{b}}$, Cyrill Bussy $^{\mathrm{c}}$, Pratap Bahadur ${ }^{\mathrm{d}}$, Sanjay Tiwari ${ }^{\mathrm{a}}{ }^{*}$ \\ ${ }^{a}$ Maliba Pharmacy College, UKA Tarsadia University, Gopal-Vidyanagar Campus, Surat, 394350, India

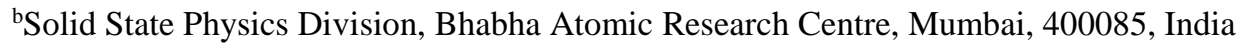 \\ 'Nanomedicine Lab, School of Biological Sciences, and Lydia Becker Institute of Immunology and Inflammation, \\ Faculty of Biology, Medicine \& Health, The University of Manchester, Manchester Academic Health Science \\ Centre, Manchester, UK; National Graphene Institute, The University of Manchester, Manchester, UK \\ ${ }^{\mathrm{d} D e p a r t m e n t ~ o f ~ C h e m i s t r y, ~ V e e r ~ N a r m a d ~ S o u t h ~ G u j a r a t ~ U n i v e r s i t y, ~ S u r a t, ~ 395007, ~ I n d i a ~}$
}

\section{ORCID numbers:}

Debes Ray: 0000-0001-5564-2973

Vinod K. Aswal: 0000-0002-2020-9026

Cyrill Bussy: 0000-0001-8870-443X

Sanjay Tiwari: 0000-0001-7898-7974

*Corresponding author tiwarisanju@gmail.com (S. Tiwari)

Tel: 91-2625-255882; Fax: 91-2625-255882 


\section{Highlights}

1. P103 micelles improve aqueous dispersibility of graphene sheets through formation of fractal aggregates.

2. Affinity of aggregates can be improved by imparting positive surface charge to the micelles.

3. Surface charge was induced in P103 micelles by incorporating DTAB, a cationic surfactant.

4. Mixed micelles triggered the formation of small-sized building blocks and improved the dispersity of sheets. 


\section{Graphical abstract}

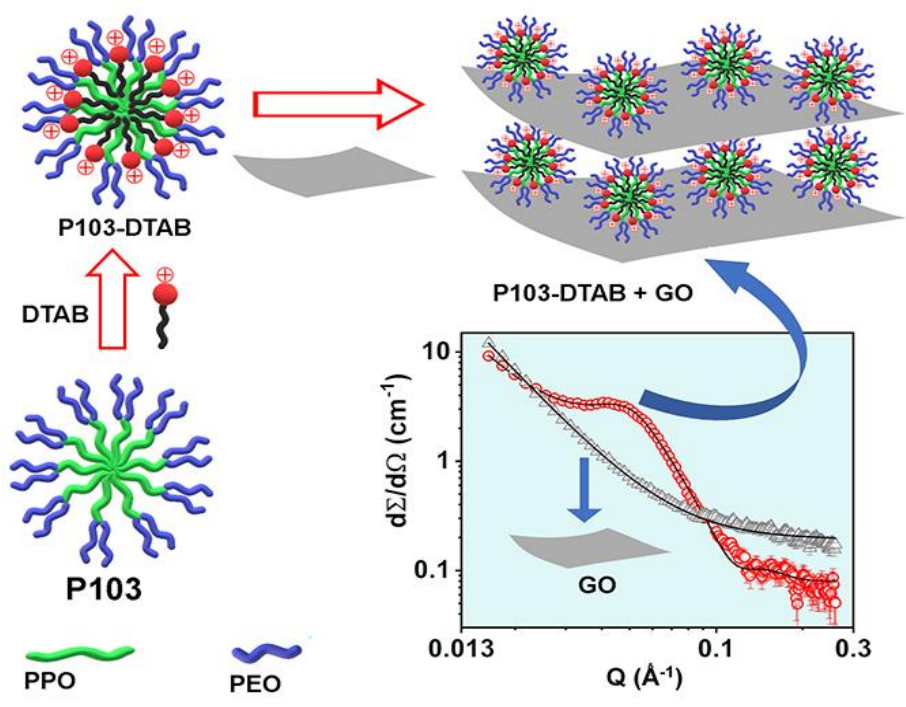




\begin{abstract}
This work reveals the role of surface charge on adsorption ability of Pluronic 103 (P103) micelles over negatively charged surface of graphene oxide (GO) nanosheets. Cationization was induced by adding dodecyltrimethylammonium bromide (DTAB, a cationic surfactant) in P103 micelles. Subsequent changes in aggregation parameters were investigated through dynamic light scattering (DLS) and small-angle neutron scattering (SANS) studies. DTAB incorporation was accompanied with a steady increase in the zeta potential and mixed micelle formation. At high surface charge density, interaction between adjacent head groups was distorted and mixed micelles dissociated. Structural developments during the adsorption of mixed micelles over sheet surface (mass fractal formation) were monitored in terms of changes in the scattering features of aggregates. These fractals emerged as a result of electrostatic interactions. Our observations indicate towards existence of small-sized building blocks at low DTAB concentration $(\leq 4 \mathrm{mM})$. Mixed micelles exhibited superior adsorption and maintained a hydration layer in the inter-sheet space. However, at higher DTAB concentration $(\geq 10 \mathrm{mM})$, micelles dissociated to produce unimers and loose aggregates. At this point, sheets exhibited quick aggregation in spite of fractal formation.
\end{abstract}

Keywords: Graphene oxide; Block copolymers; Mixed micelles; Mass fractal; Aqueous dispersibility; Zeta potential 


\section{Introduction}

Graphene oxide (GO) sheets exhibit negatively-charged carboxyl groups, polar hydroxyl groups, and hydrophobic graphitic patches. Depending upon specific method, sheets display heterogeneity in terms of layer number and interlayer structure ${ }^{1-3}$. Therefore, in spite of dense oxygen groups at the surface, high specific area and van der Waals (vdW) attractions result into formation of irreversible aggregates ${ }^{4}$. The preventive approach largely relies on the use of amphiphilic block copolymers and surfactants for stabilizing the sheets via steric and electrostatic/electrosteric repulsions. As a result of noncovalent nature of these interactions, material properties of sheets remain unharmed ${ }^{2,5}$.

In pre-micelle concentration regime, hydrophobic segment of the block copolymer spreads over unoxidized regions of sheet surface, whereas the hydrophilic counterpart dangles into the aqueous medium ${ }^{6}$. However, as the copolymer concentration approaches critical micelle concentration (CMC), sheet surface is predominantly occupied by the micelles (mass fractal development). In a recent study, we showed that fractal aggregates of uncharged ethylene oxidepropylene oxide triblock copolymer Pluronic 103 (hereafter written as P103, CMC 0.37 wt\%) ${ }^{7}$ could sterically prevent the aggregation of sheets even following exposure to protein and electrolyte ${ }^{2}$. Other groups have used low molecular weight surfactant during the exfoliation process. Given a shorter molecular length, surfactant intercalates through the graphitic gallery and produces stable dispersions. Its nonpolar tail, polar head group, counterion and aromatic substituents can be tuned to optimize the adsorption. For instance, counterion in ionic surfactants can be substituted with aromatic ones in order to increase the hydrophobicity and reduce the $\mathrm{CMC}^{8}$.

Theoretical and experimental aspects of sheet-surfactant interactions have been studied by research groups ${ }^{9-12}$. Atomistic simulations point toward random adsorption of surfactant molecules parallel to the sheet surface. However, as the surfactant concentration increases, monolayer transforms to acquire a hemispherical shape ${ }^{12}$. Thermodynamic stability of such dispersions is dictated by the last confined layer of surfactant molecule. This implies that sheets re-aggregate and tend to form thick multilayers subsequent to desorption of surfactant from the intersheet gap ${ }^{6}$. This poses a significant challenge for drug delivery applications of graphene dispersion. Possible risks include reduction in shelf life of the preparation and agglomeration of 
sheets in physiological fluids ${ }^{2,13}$. Therefore, it is important to explore novel approaches of maintaining the dispersibility of sheets in aqueous media.

Looking at the density of carboxylic acid groups, it appears that adsorption of micellar aggregates over GO surface would improve in the presence of cationic charge. This would be facilitated by long operational range of electrostatic forces ${ }^{6}$. An analogy can be drawn from the strong influence of electrostatic forces during interaction of GO with positively charged lipid membranes ${ }^{14}$. The role of electrostatic force in self-assembled systems has been revealed by Das and coworkers ${ }^{15}$ using inverse micelles composed of dodecyltrimethylammonium bromide (DTAB, CMC $\sim 15.4 \mathrm{mM})^{16}$ as a cationic surfactant. Sheets migrated to the interface in the presence of DTAB. On the contrary, interfacial localization was not observed in the anionic surfactant assembly. Moreover, loss of negatively charged groups during reductive treatment of sheets lowers the stability by augmenting vdW forces ${ }^{17}$.

It is thus interesting to evaluate the effect of surface charge in mobilizing the aggregates from the bulk to sheet surface. Herein, we have studied the contribution of electrostatic charges in sheet-micelle interactions. Cationic charge was introduced in P103 micelles via incremental addition of DTAB. Its effect was evaluated in terms of mass fractal development over GO surface. The magnitude of positive charge was restricted to low level in order to minimize the adsorption of oppositely charged species in physiological environment ${ }^{18}$. Aggregates were characterized using dynamic light scattering (DLS) and small angle neutron scattering (SANS).

\section{Materials and methods}

\section{Materials}

Graphite (>98\% purity) was purchased from Loba Chemie, India. Sulfuric acid (98\%), hydrochloric acid (35\%), sodium nitrate (98\% purity), potassium permanganate (99\% purity) and hydrogen peroxide (30\% w/v) were purchased from Rankem Laboratories, India. Solutions for small-angle neutron scattering (SANS) measurements were prepared in heavy water $(99.9 \%$ purity) procured from Tokyo Chemical Industry, India. P103 $\left(\mathrm{EO}_{17}-\mathrm{PO}_{60}-\mathrm{EO}_{17}\right)$ was received as a gift sample from BASF Corp., USA. Dodecyltrimethyl ammonium bromide (DTAB, >99\% purity) was purchased from Sigma-Aldrich, USA. All other reagents were of analytical grade and used as received. 


\section{Methods and characterizations}

GO sheets were synthesized from graphite using modified Hummers method described previously ${ }^{3,19}$. Mixed micelles (P103-DTAB) were prepared by adding different concentration of DTAB (0.1- $20 \mathrm{mM})$ to $1 \%$ solution of P103. Samples were analyzed for particle size distribution and zeta potential using Nano ZS, Malvern Instruments, UK. GO-micelle interactions were examined after adding a pre-weighed amount of GO $(1 \mathrm{mg} / \mathrm{mL})$ into micellar solutions.

Small-angle neutron scattering (SANS) experiments were performed at Dhruva Reactor, Bhabha Atomic Research Centre, India. Measurements were performed after equilibrating the system at $30^{\circ} \mathrm{C}(1 \mathrm{~h})$. The mean wavelength of the monochromatized beam was $5.2 \AA$ with a spread of $\Delta \lambda / \lambda \sim 15 \%$. Angular distribution of scattered neutrons was recorded using a onedimensional $\mathrm{He}^{3}$ position-sensitive detector. Data fitting and analyses were performed using the equations described in supporting information. Changes in dispersity were examined after adding DTAB into P103-GO dispersion.

\section{Results and discussion}

The study was divided into two parts, $(\boldsymbol{a})$ development of cationized (P103-DTAB) micelles displaying unimodal size distribution, and $(\boldsymbol{b})$ investigation on the effect of positive charge upon adsorption of micelles over sheet surface. Accordingly, we first investigated the effect of DTAB incorporation on micellar characteristics of nonionic P103. The apparent hydrodynamic diameter $\left(\mathrm{D}_{\mathrm{h}}\right)$ of 1\% P103 micelles at room temperature was recorded as $17 \mathrm{~nm}$ (polydispersity index <0.2) (Fig. 1A). It is also clear from the size distribution plots that progressive addition of DTAB caused a reduction in micelle size and favoured micelle-tounimer transition. At DTAB concentration $>10 \mathrm{mM}$, unimers and large clusters were formed as a result of destabilization of micelles ${ }^{20}$. A unimodal peak can be detected until $10 \mathrm{mM}$ DTAB. At higher concentrations, the primary peak underwent broadening and/or splitting into two or three populations. The peak at $\approx 200 \mathrm{~nm}$ represents loosely bound aggregates, whereas another one $(2-3 \mathrm{~nm})$ is attributable to unimers ${ }^{21}$. In order to ascertain that these structures originated exclusively from P103-DTAB mixture, we performed the DLS of neat DTAB (10, 15 and 20 $\mathrm{mM}$ ) solution. Alone, it did not show any such structure until $20 \mathrm{mM}$. Hence, it follows that aggregation of P103 was indeed altered in the presence of DTAB. Earlier, supramolecular structures were detected in F88 solution upon incorporation of cetyl trimethyl ammonium 
bromide (CTAB), a cationic surfactant with four carbon longer chain (C16) length. Authors argued that the hydrophobic chain avoided unfavorable contact with aqueous bulk by occupying the micelle core. Charged head groups were retained at the core-corona interface, and imparted positive surface charge to the micelles ${ }^{22}$.

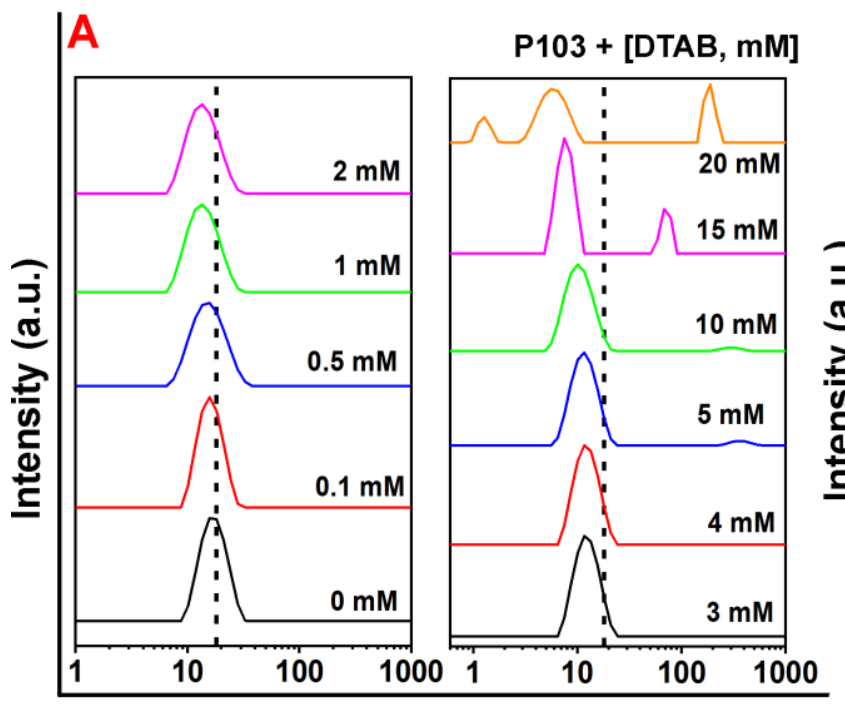

Hydrodynamic diameter $(\mathrm{nm})$

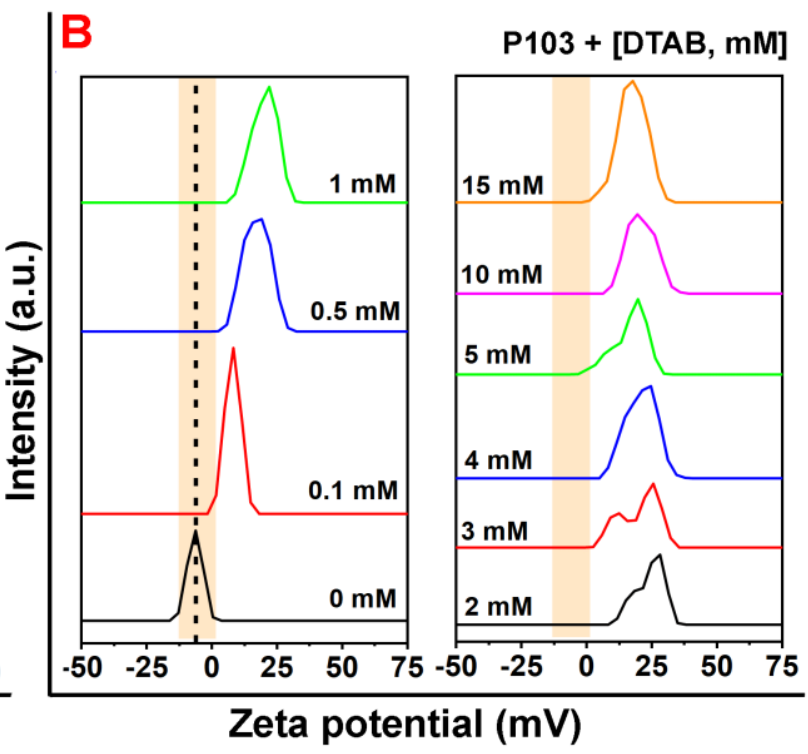

Zeta potential $(\mathrm{mV})$

Figure 1: Changes in hydrodynamic diameter and zeta potential of P103 micelles upon DTAB addition. The data were acquired at room temperature.

Micelle contraction at low DTAB concentration $(<10 \mathrm{mM})$ can be attributed to its hydrophobic engagement with PPO segment in micelle core. Micelles remained stable likely because of a balance between hydrophobic association and repulsive forces at the interface. However, at higher DTAB concentration, there would have been corresponding increase in charge density. It is important to mention that surface charge density of such systems depends on aggregation number $\left(N_{\text {agg }}\right)$ of ionic surfactants and size of the aggregates. Smaller aggregates composed of surfactants with higher $N_{\text {agg }}$ possess higher charge density ${ }^{23}$. With its low critical packing parameter $(<1 / 2)$, DTAB enhances the effective head group area in mixed micelles by virtue of its conical shape and charged headgroups ${ }^{24}$. This may deregulate the interface to the point of breakage.

Our argument on surface charge-dependent assembly of micelles is well-supported from zeta potential (ZP) measurements (Fig. 1B). Devoid of ionizable groups, P103 micelles exhibited near-zero ZP (-3 mV). It steadily shifted to positive side as the concentration of DTAB was increased. This signifies the alteration in local Coulomb forces and emergence of surface charge 
on micelle surface ${ }^{25}$. An opposite trend is observed during neutralization of surface charge ${ }^{26}$. In the Smoluchowski limit, ZP of the aggregate scales linearly with the surface charge density and is indirectly proportional to the area occupied by the charged functional group ${ }^{27}$. Given that the latter remains constant in our case, increase in $\mathrm{ZP}$ is indicative of changes in charge density. At higher concentration, DTAB caused substantial peak broadening and subsequent splitting in the distribution plot. For example, two distinct peaks $(+10.7$ and $+21 \mathrm{mV})$ can be seen with $3 \mathrm{mM}$ DTAB. However, zeta potential became constant beyond 3 mM DTAB. Altogether, DLS studies clarify that positive charge over P103 micelles can be achieved through inclusion of DTAB.

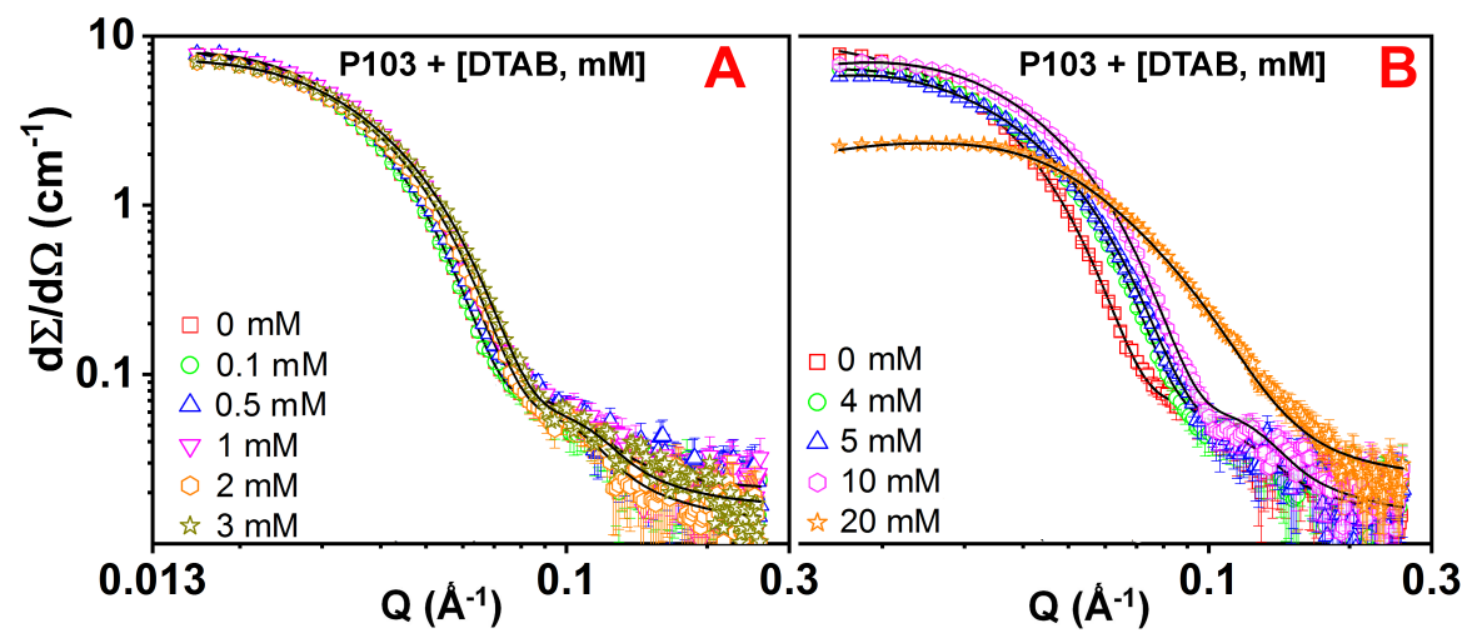

Figure 2: Changes in SANS scattering profile of P013 micelles upon DTAB addition. Shown are the data for DTAB concentration ranging from $0.1-3 \mathrm{mM}(\mathbf{A})$ and $3-20 \mathrm{mM}(\mathbf{B})$. The scattering data were fitted to sphere core-shell model.

SANS data shed more light on structural features of P103 micelles upon DTAB incorporation. The experimental data were fitted to spherical core-shell model. In order to better describe the influence of DTAB, we have classified the results in low $(<4 \mathrm{mM})$, moderate $(4-10$ $\mathrm{mM})$ and high $(\geq 10 \mathrm{mM})$ concentration regimes. To begin with, spherical shape of neat P103 micelle can be confirmed from the bell shape of its pair-distance distribution function, $p(r)$ (Fig. S1, ESI). P103-DTAB data show no noticeable change in the scattering profile of micelles in low DTAB regime (Fig. 2A). At moderate concentration, DTAB caused reduction in scattering intensity and shift of correlation peak to higher $Q$ region (Fig. 2B). Looking at the calculated structural parameters (Table 1), we suggest that reduction in scattering intensity originated from the contraction of micelle core along with lowering in aggregation number $\left(N_{\text {agg }}\right)$ of P103. As a 
result the number density of micelles increased up to five-fold. The numerical value of scattering length density highlights the contrast existing among core, shell and dispersion medium (heavy water) during data acquisition ${ }^{28}$.

Table 1: SANS-derived structural parameters of P103 micelles at increasing concentration of DTAB.

\begin{tabular}{|c|c|c|c|c|c|c|c|c|c|c|}
\hline \multirow{2}{*}{$\begin{array}{l}\text { DTAB } \\
(\mathrm{mM})\end{array}$} & \multirow{2}{*}{$\begin{array}{l}R_{c} \\
(\mathbf{n m})\end{array}$} & \multirow{2}{*}{$\begin{array}{l}\boldsymbol{R}_{h s} \\
(\mathbf{n m})\end{array}$} & \multirow{2}{*}{$\begin{array}{l}\alpha \\
(e s u)\end{array}$} & \multirow[b]{2}{*}{$\phi$} & \multirow[t]{2}{*}{ PD } & \multicolumn{2}{|c|}{$N_{\text {agg }}$} & \multirow{2}{*}{$\begin{array}{l}\mathrm{N} \times 10^{16} \\
\left(\mathrm{~cm}^{-1}\right)\end{array}$} & \multicolumn{2}{|c|}{$\operatorname{SLD}\left(\times 10^{10} \mathrm{~cm}^{-2}\right)$} \\
\hline & & & & & & P103 & DTAB & & Core & Shell \\
\hline 0.0 & 6.1 & - & - & - & 0.16 & 166 & 0 & 0.389 & 0.32 & 0.63 \\
\hline 0.1 & 6.1 & - & - & - & 0.17 & 165 & 8 & - & 0.32 & 0.63 \\
\hline 0.5 & 5.9 & - & 22.34 & - & 0.17 & 148 & 37 & - & 0.31 & 0.62 \\
\hline 1.0 & 5.7 & - & 26.04 & - & 0.17 & 133 & 66 & - & 0.31 & 0.60 \\
\hline 2.0 & 5.5 & - & 24.18 & - & 0.17 & 124 & 123 & - & 0.30 & 0.58 \\
\hline 3.0 & 5.3 & - & 15.47 & - & 0.18 & 109 & 162 & - & 0.29 & 0.55 \\
\hline 4.0 & 5.2 & - & 21.13 & - & 0.17 & 101 & 201 & - & 0.28 & 0.53 \\
\hline 5.0 & 4.9 & 7.4 & 24.61 & 0.03 & 0.19 & 87 & 215 & 1.65 & 0.27 & 0.51 \\
\hline 10.0 & 4.7 & 8.5 & 20.98 & 0.05 & 0.18 & 77 & 380 & 2.00 & 0.24 & 0.42 \\
\hline 20.0 & 2.9 & 5.4 & 12.19 & 0.06 & 0.35 & 18 & 174 & 8.87 & 0.20 & 0.32 \\
\hline
\end{tabular}

$R_{c}$ - core radius, $R_{h s}$ - hard sphere radius, $\boldsymbol{\alpha}$ - effective charge, $\phi$ - volume fraction, PD polydispersity, $N_{a g g}$ - aggregation number, $N$ - Number density of micelles, SLD - scattering length density

In nonionic assemblies, contraction of core and lowering in $N_{\text {agg }}$ at fixed amphiphile concentration have been linked with increment in number density ${ }^{29}$. Peak shift in high $Q$ region might have occurred because of appearance of additional micelles and reduction in the intermicellar distance. In spite of this, micelles did not aggregate most likely as a result of electrostatic charge imparted by DTAB molecules. As stated before, charge density of aggregates would lean over $N_{a g g}$ of DTAB and the size of the mixed micelles. This implies that higher $N_{a g g}$ of DTAB would be conducive to higher effective charge $(e)$ and greater separation among the micelles ${ }^{30}$. Formation of aggregates at 15 and $20 \mathrm{mM}$ DTAB can be explained as follows. Accommodative ability of P103 micelles saturated at high CTAB concentration alongside charge build up in shell region ${ }^{25}$. As a result, micelles were destabilized to form unimers and/or loose clusters which can clearly be noticed in DLS data (Fig. 1A). 


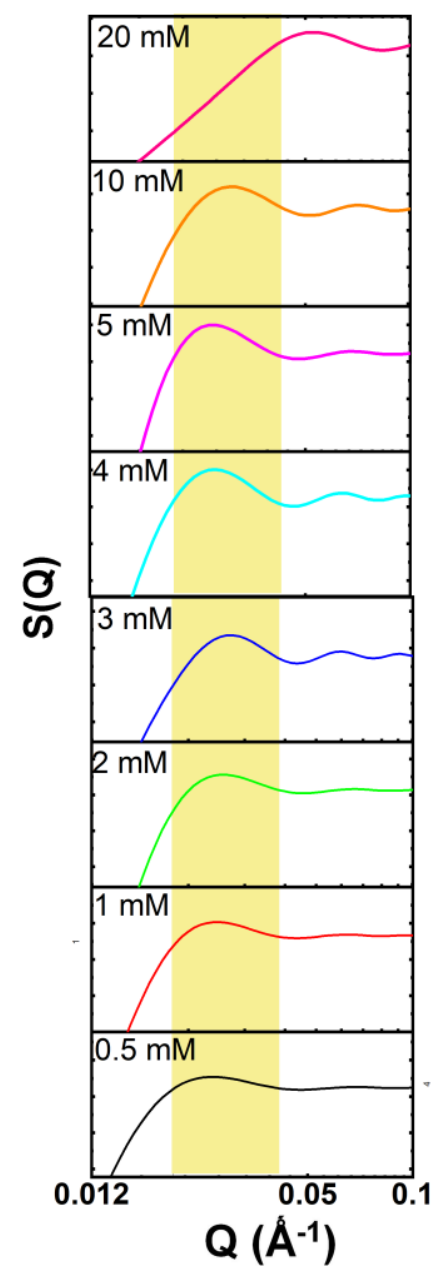

Figure 3: Changes in $S(Q)$ of P103 micelles upon DTAB addition. The numerical value on Y-axis was held identical in order to present the changes clearly.

Next, we obtained $S(Q)$ after dividing the scattering contribution of mixed aggregates with that of bare P103 micelles. Inter-particle structure factor $[S(Q)=2 \pi / \mathrm{d}]$ depends inversely on the inter-micellar distance ${ }^{30-31}$. This function is governed by a pronounced correlation peak and signifies micelle-micelle interaction. For interacting micelles, we modeled $S(Q)$ factors the Hayter-Penfold system ${ }^{30}$. As against absence of $S(Q)$ upto $2 \mathrm{mM}$, its appearance can clearly be noticed after $3 \mathrm{mM}$ DTAB. As we shifted to higher DTAB level, the increase in the peak height can be noticed (Fig. 3). It corroborates our hypothesis of the formation of small-sized micelles (high number density) which reduced the inter-micellar separation. Aggregation of micelles was obviated by the appearance of effective charge (Table 1). The changes in effective charge are in agreement to the zeta potential of aggregates (Fig. 1B). 
With these observations, it appears that cationization of P103 micelles can be achieved with the use of $3 \mathrm{mM}$ DTAB without triggering unimerization or loose cluster development. Hence, we investigated the adsorption ability of P103-DTAB micelles over GO sheets. A comparison between neutron scattering profile of sheets in neat P103 and DTAB is shown in Fig. 4. Whereas P103 micelles fitted to spherical core-shell model, scattering data of GO powder showed a good fit to mass fractal model. In agreement to our earlier work ${ }^{32}$, scattering data of P103-GO fitted to a combination of spherical core-shell and mass fractal models. Apart from shift in scattering to high $Q$ region, sheet incorporation was accompanied with hump formation (Fig. 4A). Other noteworthy observations include reduction of aggregation number (41 from 166), shrinkage of micelles (3.8 from $6.1 \mathrm{~nm}$ ) and more than sixty-fold increase in the number density of micelles (Table TS1, ESI). We speculate that additional small sized micelles covered the sheet surface and formed the fractal structure. The hump in scattering profile of P103-GO dispersion can be ascribed to the structure factor that emerged as a result of micelle-sheet interaction. We believe that the micelles are either adsorbed over the surface or trapped between the sheets ${ }^{5,32}$.
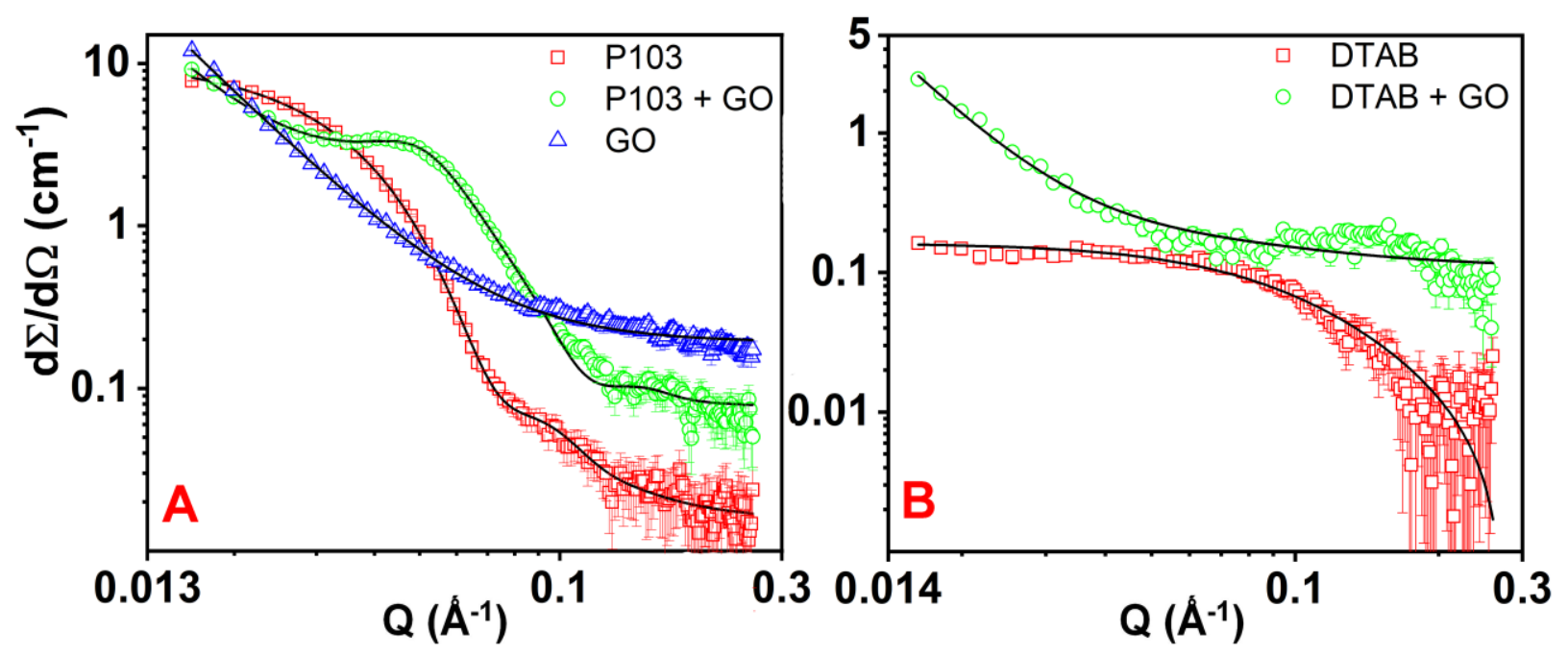

Figure 4: Neutron scattering profile of P103 micelles upon GO addition. The data of P103 micelles were fitted to spherical core-shell model. GO powder fitted best with mass-fractal model. P103-GO dispersion was fitted with a combination of core-shell sphere and mass-fractal models (A). Scattering profiles of DTAB $(20 \mathrm{mM})$ and DTAB-GO are shown in (B). DTAB dispersion was fitted with ellipsoid core-shell model. DTAB-GO dispersion was fitted with a combination of ellipsoid core-shell and mass-fractal models. 
We observed ellipsoidal micelles in the case of pure DTAB $(20 \mathrm{mM})$ solution (Fig. 4B). Acquired data fitted well to ellipsoidal core-shell model. Semi-major and semi-minor axis of micelle was found to be 2.17 and $1.9 \mathrm{~nm}$. These micelles were adsorbed over GO surface and produced fractal structures with 2.85 fractal dimension (Table TS2, ESI). The latter is verifiable from the fit of scattering data to ellipsoidal core-shell and mass fractal combination. Apparently, the adsorption was driven through electrostatic attraction between negatively charged sheet surface and cationic DTAB head group ${ }^{9,15}$. In spite of this, the dispersion showed poor stability suggesting that fractal development cannot be an absolute indicator of higher dispersibility. It, thus, poses a question on the ability of structures in effectively masking the effect of hydrophobic patches over sheet surface ${ }^{3}$.

McCoy et al. ${ }^{5}$ argued that cationic unimers adsorb via head groups which pushes the hydrophobic tail into bulk aqueous medium. Entropic factors allow the alignment of another surfactant molecule in anti-parallel orientation. Placement of head groups in opposite ('headout') direction creates energetically favorable tail-tail association. However, as the local crowding occurs, unimer adsorption is hindered because of two factors; (a) compensation of surface charge among vertically arranged surfactant units, and (b) shielding of surface in the event of lateral stretching of surfactant tail ${ }^{33}$. In such a situation, desirable aqueous dispersity may not be achieved in spite of surfactant adsorption. In our case, it was evident from precipitation of sheets containing $10 \mathrm{mM}$ DTAB within $4 \mathrm{~h}$ (data not shown).

Next, we have addressed the issue of sheet stabilization with charged P103-DTAB assemblies. Acquired SANS data have been fitted using the combination of mass fractal and spherical core-shell model. In this data, no features corresponding to the overall size of aggregates (lower $Q$ cut-off) within the $Q$ range of the present measurements were observed. Therefore, we used a fixed overall size greater than $2 \pi / Q_{\min }$ for the analysis ${ }^{34}$. A comparison between the scattering profile of P103 and P103-DTAB micelles highlights that role of positive change upon aggregate adsorption on sheets (Fig. 5, A-B). Improvement in dispersibility of inorganic nanoparticles has been investigated in terms changes in fractal dimension, type of building block, radius of aggregates and the overall size of fractal aggregates ${ }^{35-36}$. Calculation of these parameters could not be performed in our case because of co-existance of multiple structures (mixed micelles, unimers, hemi-micelles, etc). Therefore, we have used the summation model (Fig. S2-S4, ESI) for fitting the scattering data. Data fitting involved mass fractal model 
for low $Q$ region and sphere core shell model (with Hayter Penfold structure factor) for mid- and high $Q$ region data ${ }^{5}$. In order to understand the changes comprehensively, we spllitted the scattering profile into low (0.016-0.037 ̊́), middle (0.038-0.059 ̊́) and high $Q$ (0.060-0.1 ̊́) regions. Scattering at low- and high $Q$, respectively, is controlled by the fractal aggregates and micelles. On the other hand, middle $Q$ region is contributed by the building block (P103$\mathrm{DTAB} / \mathrm{GO}$ ) of aggregate ${ }^{36}$. Existence of fractal structures in low $Q$ region can be confirmed from a linear scattering profile ${ }^{37}$. As stated earlier, these fractals would have developed either through micelle adsorption over sheet surface (hump-like build up in middle $Q$ ) or following their confinement between the sheets (higher scattering in low $Q)^{34}$.

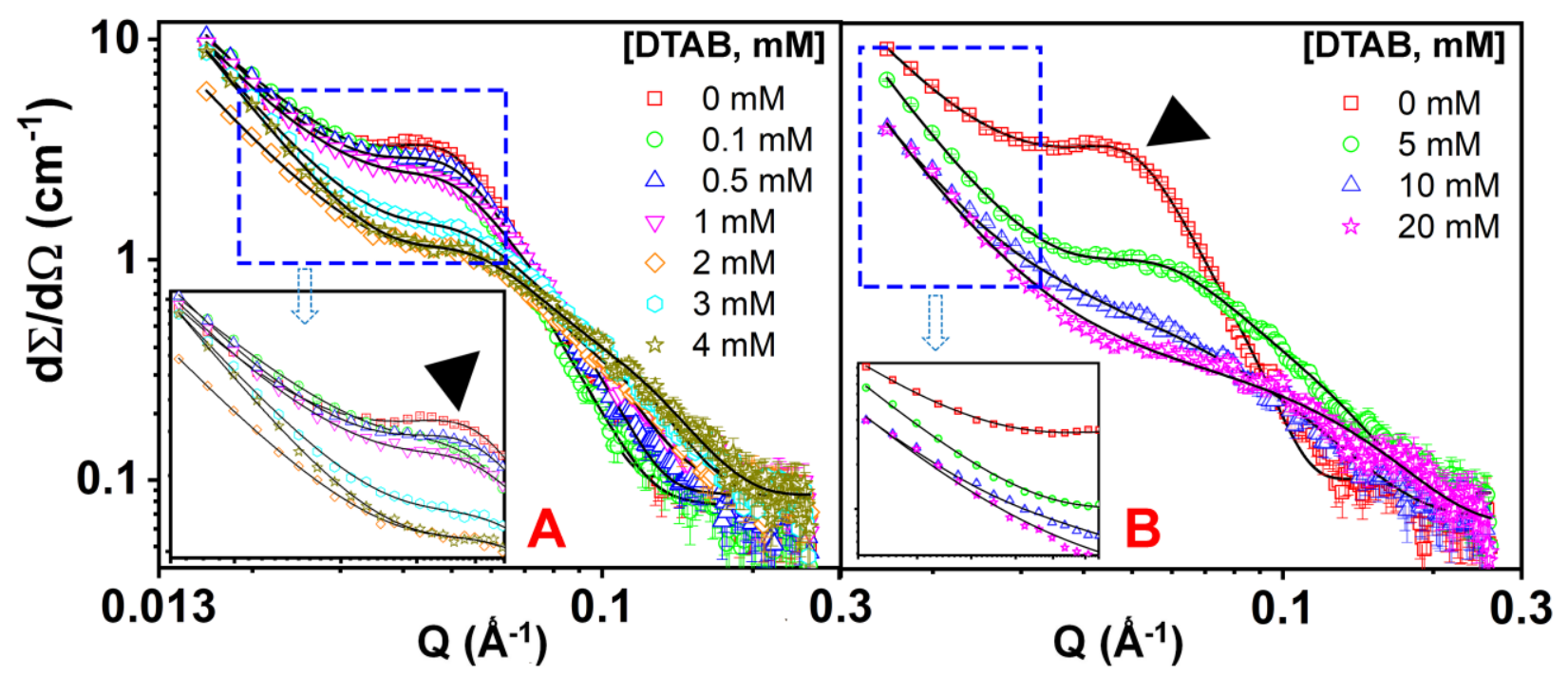

Figure 5: Changes in neutron scattering profile of P103-GO dispersion with varying concentrations of DTAB. Scattering data till $4 \mathrm{mM}$ DTAB (micelle dissociation point) (A) and post-micelles breakage (B). The data were fitted with spherical core-shell and mass fractal models.

Increase in the width of scattering build-up during DTAB addition signifies enhancement in the attractive interaction among sheets and mixed micelles. Intriguingly, as DTAB concentration increased, the hump underwent suppression $(5 \mathrm{mM})$ and complete disappearance $(\geq 10 \mathrm{mM}$; Fig. 5, A-B). This is indicative of disruption in aggregate-sheet interaction beyond a threshold DTAB concentration (4 mM). In addition, the data highlight another important point; pre-mature disruption of micelles in the presence of sheets. This was confirmed by substracting the contribution of mass fractal scattering from the fitted data (P103-DTAB+GO). Here, the fitted line represents the sphere model along with Hayter Penfold structure factor (Fig. S5). It appears that, responding to sheet's hydrophobicity, surfactant assemblies were depleted from the 
bulk solution in order to cover the hydrophobic points ${ }^{5}$. The involvement of electrostatic interaction between sheet and mixed micelles can be verified from the drop in zeta potential (8$12 \mathrm{mV}$ ) of the dispersion. Moreover, drop in CMC highlights the changes in hydrophobicity of P103-DTAB in association with sheets (Figs. S6-S7). Looking together the changes in size of P103 micelles during DTAB incorporation and the affinity of P103-DTAB micelles for sheet surface, changes in the size of fractal aggregates can be expected ${ }^{38-39}$. Accordingly, we have presented a plausible mechanism for the formation of small sized compact building blocks.
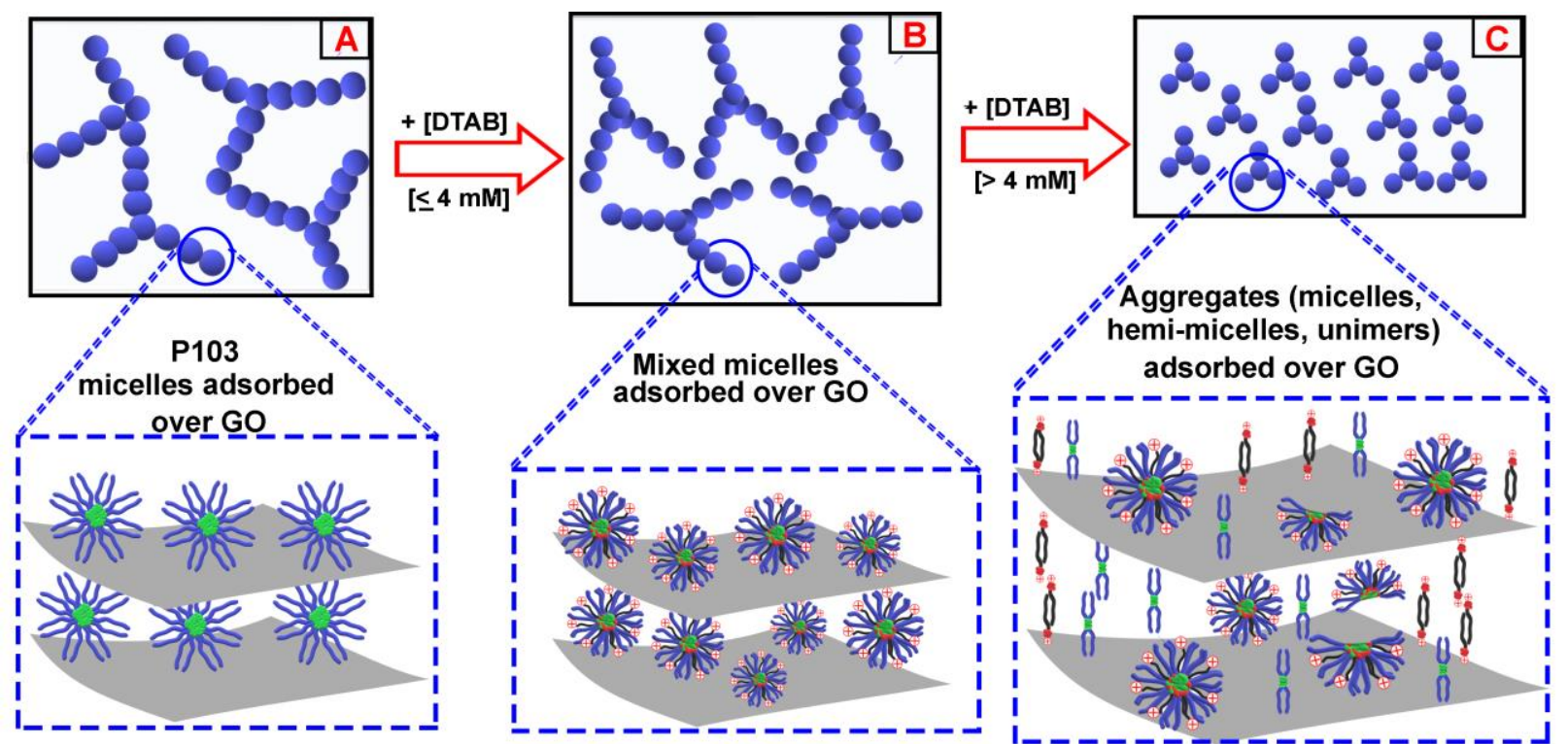

Figure 6: Scheme showing mechanism of sheet stabilization through adsorption of aggregates. Initially, P103 micelles occupied the surface and formed fractal aggregates (A). Later, fractal dimension and building block radius may vary in accordance to DTAB concentration. At low concentration $(\leq 4 \mathrm{mM})$, surface is covered by cationic mixed micelles in high number density. Building block radius of aggregates (per fractal structure) would decrease in this regime. Eventually, overall size of fractal aggregates would reduce thereby leading to emergence of densely packed structures in the medium. At this point, aqueous dispersity improves because of high affinity between negatively charged sheets and cationic aggregates (B). However, as DTAB concentration becomes high ( $\geq 4 \mathrm{mM})$, sheet surface would be dominated by unimers and loose assemblies derived from the dissociated micelles $(\mathbf{C})$. The steric barrier would break and sheets tend to aggregate.

We assume that micelle adsorption would minimize the inter-sheet interaction and increase the interlayer distance (Fig. 6A). Given a highly hydrated corona, it is expected that a sub-nanometer thick hydration layer would be maintained over sheet surface ${ }^{40}$. The minimum inter-sheet distance in such surface-separated structures would be equivalent to micelle diameter 
$(8.5$ to $10 \mathrm{~nm})$. This is analogous to a mechanism of sheet stabilization demonstrated by Lambert's group ${ }^{41}$ employing pyrene-oligoethylene glycol-based surfactants. Atomically flat sheets were initially occupied by planar starfish micelles. At higher concentration, surfactant molecules clustered to form discrete conical structures which eventually acted as inter-sheet barrier.

An important finding is related to reduction in the building block (sheets covered by mixed micelles) radius at moderate DTAB concentration $(\leq 4 \mathrm{mM})$. We hypothesize that in this concentration regime, DTAB would reduce the number of building blocks per fractal structure. It would lead to increase in the number density of small-sized fractal structures (in sync with increased number density of micelles, Table 1). Reduction in fractal size is verifiable from the scattering build-up at the low $Q$ region in Fig. $5^{36}$. Eventually, the number density of fractals contributes to higher fractal dimension. However, at high concentration (>10 mM, for instance), DTAB probably destabilized the aggregates into unimers, clusters or hemi-micelles exhibiting low affinity for the sheets (Fig. 6, B-C). We qualitatively verified that superior aqueous dispersibility of sheets was attained with mixed micelles containing <4 mM DTAB.

\section{Conclusion}

In this work, the adsorption affinity of cationic mixed micelles (P103-DTAB) over anionic GO sheets has been investigated as a function of DTAB addition. Incorporation of DTAB correlated with the appearance of positive charge over P103 micelles until the point of de-micellization. Our observations reveal that charged micelles were adsorbed over sheets via electrostatic attraction and produced fractal structures. These aggregates acted as inter-sheet barrier while engaging with the dispersion medium. The effect of DTAB addition was clearly visible in fractal structures. At low concentration $(\leq 4 \mathrm{mM})$, it stimulated formation of smaller micelles at high number density which were effectively adsorbed over the sheets to produce dense building blocks. Such an arrangement minimizes the inter-sheet interactions. However, the building blocks became loose as the concentration of DTAB was increased beyond $4 \mathrm{mM}$. It will be interesting to study the stability of this dispersion in physiological fluids.

\section{Authorship contribution statement}

Rahul Patil: Investigation, Writing - original draft. Debes Ray: Formal analysis. Vinod K. Aswal: Formal analysis. Cyrill Bussy: Writing - review \& editing. Pratap Bahadur: Writing - 
review \& editing. Sanjay Tiwari: Conceptualization, Writing - review \& editing, Project administration.

\section{Declaration of competing interest}

Authors declare no conflict of interest.

\section{Acknowledements}

ST thanks the Science \& Engineering Research Board (SERB), New Delhi, for funding under ECRA scheme (\# ECR/2017/000903). DLS facility at Maliba Pharmacy College is funded by DST-FIST, New Delhi. 


\section{References}

1. Lin, S.; Shih, C. J.; Strano, M. S.; Blankschtein, D., Molecular insights into the surface morphology, layering structure, and aggregation kinetics of surfactant-stabilized graphene dispersions. J. Am. Chem. Soc. 2011, 133, 12810-12823.

2. Patil, R.; Bahadur, P.; Tiwari, S., Dispersed graphene materials of biomedical interest and their toxicological consequences. Adv. Colloid Interface Sci. 2020, 275, 102051.

3. Patil, R.; Patel, H.; Pillai, S. B.; Jha, P. K.; Bahadur, P.; Tiwari, S., Influence of surface oxygen clusters upon molecular stacking of paclitaxel over graphene oxide sheets. Mater. Sci. Eng. C 2020, 116, 111232.

4. Nazari, B.; Ranjbar, Z.; Moghaddam, A.R.; Momen, G.; Ranjbar, B., Dispersing graphene in aqueous media: Investigating the effect of different surfactants. Colloids Surf. A 2019, 582, 123870 .

5. McCoy, T. M.; De Campo, L.; Sokolova, A. V.; Grillo, I.; Izgorodina, E. I.; Tabor, R. F., Bulk properties of aqueous graphene oxide and reduced graphene oxide with surfactants and polymers: adsorption and stability. Phys. Chem. Chem. Phys. 2018, 20, 16801-16816.

6. Hong, B. J.; Compton, O. C.; An, Z.; Eryazici, I.; Nguyen, S. T., Successful stabilization of graphene oxide in electrolyte solutions: enhancement of biofunctionalization and cellular uptake. ACS Nano 2012, 6, 63-73.

7. Fernandez, V.; Soltero, J.; Puig, J.; Rharbi, Y., Temporal evolution of the size distribution during exchange kinetics of pluronic P103 at low temperatures. J. Phys. Chem. B 2009, 113, 30153023.

8. Mohamed, A.; Ardyani, T.; Bakar, S. A.; Sagisaka, M.; Umetsu, Y.; Hamon, J.; Rahim, B. A.; Esa, S. R.; Khalil, H. A.; Mamat, M. H., Rational design of aromatic surfactants for graphene/natural rubber latex nanocomposites with enhanced electrical conductivity. J. Colloid Interface Sci. 2018, 516, 34-47.

9. Meng, W.; Gall, E.; Ke, F.; Zeng, Z.; Kopchick, B.; Timsina, R.; Qiu, X., Structure and interaction of graphene oxide-cetyltrimethylammonium bromide complexation. J. Phys. Chem. C 2015, 119, 21135-21140.

10. Cao, J.; Bai, X.; Ye, Z.; Chen, W.; Ge, H.; Ding, Y.; Hua, Z., Enhanced Transport of TiO2Reduced Graphene Oxide Nanocomposites in Saturated Porous Media: the Impact of Loaded TiO2 Shape and Solution Conditions. Water Air Soil Pollut. 2020, 231, 1-11.

11. Ardyani, T.; Mohamed, A.; Bakar, S. A.; Sagisaka, M.; Umetsu, Y.; Mamat, M. H.; Ahmad, M. K.; Khalil, H. A.; King, S.; Rogers, S. E., Surfactants with aromatic headgroups for optimizing properties of graphene/natural rubber latex composites (NRL): Surfactants with aromatic amine polar heads. J. Colloid Interface Sci. 2019, 545, 184-194.

12. Poorsargol, M.; Alimohammadian, M.; Sohrabi, B.; and Dehestani, M., Dispersion of graphene using surfactant mixtures: Experimental and molecular dynamics simulation studies. Appl. Surf. Sci. 2019, 464, 440-450.

13. Huang, C.; Wu, J.; Jiang, W.; Liu, R.; Li, Z.; Luan, Y., Amphiphilic prodrug-decorated graphene oxide as a multi-functional drug delivery system for efficient cancer therapy. Mater. Sci. Eng. C 2018, 89, 15-24.

14. Frost, R.; Jonsson, G. E.; Chakarov, D.; Svedhem, S.; Kasemo, B., Graphene oxide and lipid membranes: interactions and nanocomposite structures. Nano Lett. 2012, 12, 3356-3362.

15. Das, K.; Maiti, S.; Ghosh, M.; Mandal, D.; Das, P. K., Graphene oxide in cetyltrimethylammonium bromide (CTAB) reverse micelle: a befitting soft nanocomposite for improving efficiency of surface-active enzymes. J. Colloid Interface Sci. 2013, 395, 111-118. 
16. Bergström, L. M.; Aratono, M., Synergistic effects in mixtures of two identically charged ionic surfactants with different critical micelle concentrations. Soft Matter 2011, 7 (19), 88708879.

17. Gudarzi, M. M., Colloidal stability of graphene oxide: aggregation in two dimensions. Langmuir 2016, 32, 5058-5068.

18. Sharma, R.; Baik, J. H.; Perera, C. J.; Strano, M. S., Anomalously large reactivity of single graphene layers and edges toward electron transfer chemistries. Nano Lett. 2010, 10 , 398-405.

19. Patil, R.; Kansara, V.; Ray, D.; Aswal, V. K.; Jha, P. K.; Bahadur, P.; Tiwari, S., Slow degrading hyaluronic acid hydrogel reinforced with cationized graphene nanosheets. Int. J. Biol. Macromol. 2019, 141, 232-239.

20. Thummar, A. D.; Sastry, N. V.; Verma, G.; Hassan, P. A., Aqueous block copolymersurfactant mixtures - Surface tension, DLS and viscosity measurements and their utility in solubilization of hydrophobic drug and its controlled release. Colloid Surf. A 2011, 386, 54-64.

21. Parmar, A.; Chavda, S.; Bahadur, P., Pluronic-cationic surfactant mixed micelles: Solubilization and release of the drug hydrochlorothiazide. Colloid Surf. A 2014, 441, 389-397.

22. Singh, P. K.; Kumbhakar, M.; Ganguly, R.; Aswal, V. K.; Pal, H.; Nath, S., Time-resolved fluorescence and small angle neutron scattering study in pluronics-surfactant supramolecular assemblies. J. Phy. Chem. B 2010, 114 , 3818-3826.

23. Hu, C.; Li, R.; Yang, H.; Wang, J., Properties of binary surfactant systems of nonionic surfactants C12E10, C12E23, and C12E42 with a cationic gemini surfactant in aqueous solutions. J. Colloid Interface Sci. 2011, 356, 605-613.

24. Vicente, F. A.; Cardoso, I. s. S.; Sintra, T. n. E.; Lemus, J.; Marques, E. F.; Ventura, S. n. P.; Coutinho, J. o. A., Impact of surface active ionic liquids on the cloud points of nonionic surfactants and the formation of aqueous micellar two-phase systems. J. Phys. Chem. B 2017, 121, 8742-8755.

25. Nambam, J.; Philip, J., Effects of interaction of ionic and nonionic surfactants on selfassembly of PEO-PPO-PEO triblock copolymer in aqueous solution. J. Phys. Chem. B 2012, 116, 1499-1507.

26. Kihara, S.; van der Heijden, N. J.; Seal, C. K.; Mata, J. P.; Whitten, A. E.; Koper, I.; McGillivray, D. J., Soft and hard interactions between polystyrene nanoplastics and human serum albumin protein corona. Bioconjug. Chem. 2019, 30, 1067-1076.

27. Vogel, R.; Pal, A. K.; Jambhrunkar, S.; Patel, P.; Thakur, S. S.; Reategui, E.; Parekh, H. S.; Saa, P.; Stassinopoulos, A.; Broom, M. F., High-resolution single particle zeta potential characterisation of biological nanoparticles using tunable resistive pulse sensing. Sci. Rep. 2017, 7,17479 .

28. Griffiths, P. C.; Paul, A.; Khayat, Z.; Heenan, R. K.; Ranganathan, R.; Grillo, I., A smallangle neutron scattering study of biologically relevant mixed surfactant micelles comprising 1, 2diheptanoyl-sn-phosphatidylcholine and sodium dodecyl sulfate or dodecyltrimethylammonium bromide. Soft Matter 2005, 1, 152-159.

29. Pathan, H.; Patil, R.; Ray, D.; Aswal, V. K.; Bahadur, P.; Tiwari, S., Structural changes in non-ionic surfactant micelles induced by ionic liquids and application thereof for improved solubilization of quercetin. J. Mol. Liq. 2019, 290, 111235.

30. De, S.; Aswal, V. K.; Goyal, P. S.; Bhattacharya, S., Small-angle neutron scattering studies of different mixed micelles composed of dimeric and monomeric cationic surfactants. J. Phys. Chem. B 1997, 101 , 5639-5645. 
31. Mahajan, R.; Vohra, K.; Aswal, V., Small angle neutron scattering measurements of aggregation behaviour of mixed micelles of conventional surfactants with triblock polymer L64. Colloid Surf. A 2008, 326, 48-52.

32. Patil, R.; Marathe, D.; Roy, S. P.; Ray, D.; Aswal, V. K.; Jha, P. K.; Bahadur, P.; Tiwari, S., Colloidal stability of graphene oxide nanosheets in association with triblock copolymers: A neutron scattering analysis. Mater. Sci. Eng. C 2020, 109, 110559.

33. Goloub, T.P.; Koopal, L.K., Adsorption of cationic surfactants on silica. Comparison of experiment and theory. Langmuir 1997, 13, .673-681.

34. Ray, D.; Kumar, S.; Aswal, V. K.; Kohlbrecher, J., Tuning nanoparticle-micelle interactions and resultant phase behavior. Langmuir 2018, 34, 259-267.

35. Yadav, I.; Kumar, S.; Aswal, V.; Kohlbrecher, J., Small-angle neutron scattering study of differences in phase behavior of silica nanoparticles in the presence of lysozyme and bovine serum albumin proteins. Phys. Rev. E 2014, 89, 032304.

36. Kumar, S.; Aswal, V. K.; Kohlbrecher, J., Size-dependent interaction of silica nanoparticles with different surfactants in aqueous solution. Langmuir 2012, 28, 9288-9297.

37. Teixeira, J., Small-angle scattering by fractal systems. J. Appl. Crystallogr. 1988, 21, 781785.

38. Yadav, I.; Kumar, S.; Aswal, V. K.; Kohlbrecher, J., Structure and Interaction in the pHDependent phase behavior of nanoparticle-protein systems. Langmuir 2017, 33, 1227-1238.

39. Yang, B.; Lowe, J. P.; Schweins, R.; Edler, K. J., Small angle neutron scattering studies on the internal structure of poly (lactide-co-glycolide)-block-poly (ethylene glycol) nanoparticles as drug delivery vehicles. Biomacromolecules 2015, 16, 457-464.

40. Li, J.; Li, J.; Jiang, L.; Chen, X.; Luo, J., Cationic surfactant micelles lubricate graphitic surface in water. Langmuir 2019, 35, 11108-11113.

41. Robinson, B. J.; Bailey, S. W.; O'Driscoll, L. J.; Visontai, D.; Welsh, D. J.; Mostert, A. B.; Mazzocco, R.; Rabot, C.; Jarvis, S. P.; Kolosov, O. V.; Bryce, M. R.; Lambert, C., Formation of two-dimensional micelles on graphene: Multi-scale theoretical and experimental study. ACS Nano 2017, 11, 3404-3412. 